Article

\title{
Microstructure and Resistivity Analysis of Silver Nanoparticle-Based Crystalline Conductive Films Synthesized using PEG Surfactant
}

\author{
Faisal Mustafa, Muhammad Razwan and Saima Shabbir * iD \\ Department of Materials Science and Engineering, Institute of Space Technology, Islamabad 44000, Pakistan; \\ imfaisalmustafa@gmail.com (F.M.); mrazwan.ist@gmail.com (M.R.) \\ * Correspondence: saima.shabbir@ist.edu.pk
}

Received: 29 March 2019; Accepted: 23 April 2019; Published: 27 April 2019

check for updates

\begin{abstract}
Silver nanoparticle-based crystalline conductive films were synthesized using a simple and environmentally friendly method centered on chemical reduction. A stoichiometric balance of three different molecular weights of polyethylene glycol (PEG) was used as a capping agent. Resistivity, and its correlation with temperature and the particle size of nanoparticle films, was probed. The silver nanoparticles were characterized using thermogravimetric analysis (TGA) and field emission scanning electron microscopy (FESEM). Further silver films deposited on a glass substrate were characterized by FESEM, Fourier transform infrared spectroscopy (FTIR), X-ray diffraction (XRD) and resistivity measurements. Particle size distribution and room temperature electrical conductivity were also investigated. The high conductivity of sintered films suggested applications for the ink-jet printing of electronic circuitry on thermally sensitive substrates.
\end{abstract}

Keywords: silver nanoparticles; resistivity; PEG; surfactant

\section{Introduction}

Over the past decade the electronics industry has seen a dramatic reduction in size, especially relating to integrated circuits. Research in this field is geared towards size minimization along with fast and inexpensive synthetic processes. The so-called "smart materials" have become a very attractive and actively developing area in the last few years [1-23], due not only to their aesthetics but also to their ability to render devices that adapt to varying chemical and physical conditions in the environment $[8,9]$. Traditional methods for fabricating circuit films, i.e., screen printing, photolithography and electroplating, make use of etching technologies and selective masking to create regions of metallization on non-conducting substrates [21]. Such methods though effective, have the disadvantages of material wastage, chemical pollution and slow product translation. Hence, direct writing technologies are gaining more interest, where a material having desirable electronic properties is deposited over a substrate in particulate form and later converted to conductive elements.

Previously, conductive inks were synthesized using different metal precursors like carbon, silver, or copper. They were analyzed for resistivity, viscosity, stability and cost parameters. One of the major challenges, along with their cost, has been the manufacturing technique and adhesion with a substrate. Several metal nanoparticles, capping agents, reducing agents and temperatures were studied for the formulation of conductive inks. In this context, the specific characteristics of conductive nanoparticles, such as decrease in melting point with size, enhanced reactivity of surface atoms and high electric conductivity, make them useful for the fabrication of electronic components [11-13]. Due to the high electrical conductivity silver nanoparticle-based inks were considered excellent for printable electronics. However, most commercially available inks use either high boiling point solvents or toxic chemicals, which are problematic in real industrial applications [14,15]. 
In earlier studies for the preparation of silver conductive ink or films, researchers frequently used polyvinyl pyrrolidone (PVP) and polyacrylic acid (PAA) as a capping agent $[10,14,17,23]$. In some investigations PEG or PAA was incorporated but only a single molecular weight was used in the syntheses, which led to agglomeration and less conductivity [24,25]. Previously the shape and size regulation of silver nanospheres was probed by varying the molecular weight of PVP capping agent. It was established that the shortest PVP chain, with the lowest steric effect, easily shielded the silver nucleus surface but offered poor control over nanoparticle accumulation. Alternatively, PVP grades with a higher molar mass afforded efficient anti-agglomeration, thereby, inducing the development of smaller sized particles [26]. Therefore, this report of a simple, cost effective and environmentally friendly technique, using an equimolar mixture of three different molecular weights of the PEG capping agent, effectively achieved deagglomeration as well as a resistivity approaching the bulk silver. Ensuing conductive films allowed a direct relationship between temperature and conductivity. Additionally, we have devised a microscopy-based analysis technique to find the electrical resistivity of the resultant films. These films deposited over glass substrate were thermally treated at different elevated temperatures for various times. Sintered films showed good conductivities, demonstrating that their conductivity is suitable for printing electronic circuitry on thermally sensitive substrates.

\section{Experimental Section}

\subsection{Materials}

Silver nitrate $\left(\mathrm{AgNO}_{3}\right)$ purchased from British Drug Houses $(\mathrm{BDH})$ was used as a metal precursor. Deionized water and ethanol were used as solvents. Different grades of polyethylene glycol [molecular weight (MW) 4000, 6000, 8000], procured from Sigma-Aldrich, were applied as capping agents. Ethylene glycol (EG) was used as a viscosifying agent and diethanol amine (DAE, 98\%) served as a reducing agent.

\subsection{Fabrication of Silver Ink and Silver Conductive Films}

Silver nanoparticles were produced by mixing $1 \mathrm{~g}$ PEG, consisting of an equimolar ratio of three MWs, $25 \mathrm{~g}$ of deionized water and $20 \mathrm{~g}$ of DEA, and continuously stirring for $1.5 \mathrm{~h}$. Ten grams of $\mathrm{AgNO}_{3}$, dissolved in $7.5 \mathrm{~mL}$ of deionized water, was injected in the above solution and the mixture was stirred for $22 \mathrm{~h}$. Initially the color of the solution was transparent but gradually it became brownish and finally a dark blackish color appeared. Finally, the walls of the breaker obtained a silver luster. Then the solution was sonicated at $65^{\circ} \mathrm{C}$ for $1.5 \mathrm{~h}$ and after cooling it was titrated using $150 \mathrm{~mL}$ of ethanol at the rate of $5 \mathrm{~mL} / \mathrm{min}$. After titration stirring was continued for $2 \mathrm{~h}$ [10].

Later the supernatant was decanted and the precipitates were centrifuged at $4000 \mathrm{rpm}$ for $20 \mathrm{~min}$ to concentrate the sample. The ensuing mixture was then redispersed in $12 \mathrm{~mL}$ of water by vortexing and sonication at room temperature. Further centrifugation at $4000 \mathrm{rpm}$ for $20 \mathrm{~min}$ removed any excess PEG. This washing step was performed three times. The different molecular weights of PEG could not dissolve all at once during the washing process, thus allowing deagglomeration resulting in the formation of stable and uniform size nanoparticles [24-26]. In the end, EG was added to the precipitates as the viscosifying agent and the resulting solution was coated over a glass substrate.

\subsection{Characterizations}

The PEG content was determined before and after washing by TGA analysis in air from 30 to $800{ }^{\circ} \mathrm{C}$ at a rate of $10^{\circ} \mathrm{C} \mathrm{min}^{-1}$ using Mettler Toledo TGA/SDTA851 [16]. After washing the sample and confirming the presence of silver, the sample was tested via FESEM. The surface morphology of the samples was observed by FESEM (MIRA3 TESCAN) using an operating voltage of $10 \mathrm{kV}$. FESEM, equipped with EDS, was performed during the synthesis and after the silver film was formed and sintered in order to analyze the size of particles, the elemental analysis and the amount of PEG in the 
sample, as PEG may hinder the electrical properties of the ink. Particle size analysis was performed on MALVERN Master Sizer Hydro 3000 equipment using distilled water as the solvent.

Firstly, the formation and morphology of the silver nanoparticles was confirmed. Then, their size and size distributions were evaluated. FTIR was performed to investigate the major bands in the range of $500-4000 \mathrm{~cm}^{-1}$ (wavenumber). The XRD technique was performed to analyze the crystallographic structure of the conductive film. The carbon X-ray source was used, having a 1.5-1.6 ̊ wavelength. The crystallite size was obtained at full width half maximum (FWHM). The silver nanoparticles were confirmed using Powder Diffraction Standards (PDS), silver file No. 04-0783. The resistivity analysis was performed using a digital multimeter (DMM) (EDM-4760).

\section{Results and Discussion}

\subsection{Thermogravimetric Analysis (TGA)}

TGA was performed to ensure that the capping agent and other organic impurities were removed from the product because these may act as contaminants in the final sample, thus reducing the conductivity. Therefore, TGA was done before and after the washing step. The thermogram curve $\mathrm{C} 1$, before washing (Figure 1), showed too many dips, indicating the decomposition of the ethanol, polymer, water and silver nanoparticles. After washing the C2 curve (Figure 1) depicted only one dip of silver nanoparticles, indicating that the capping agent and other organic or polymeric components were completely removed and the sample comprised pure silver nanoparticles.

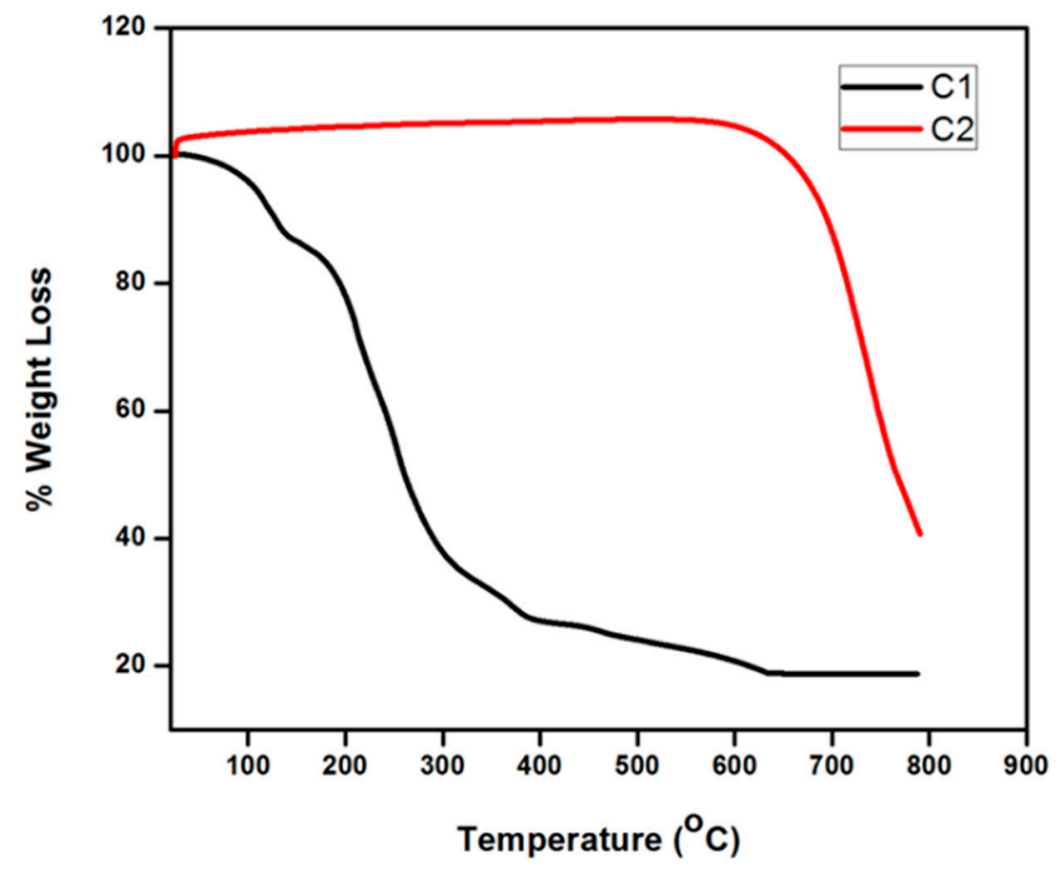

Figure 1. Thermo gravimetric Analysis (TGA) curves of Polyethylene Glycol (PEG)-capped particles with ethanol before washing $(\mathrm{C} 1)$ and after $(\mathrm{C} 2)$ washing.

\subsection{Energy Despersive X-ray Spectroscopy (EDS)}

Elemental analysis of silver nanoparticles using EDS showed the presence of silver in a greater concentration (Figure 2). EDS confirmed that the nanoparticles shown in the SEM images were Ag. 


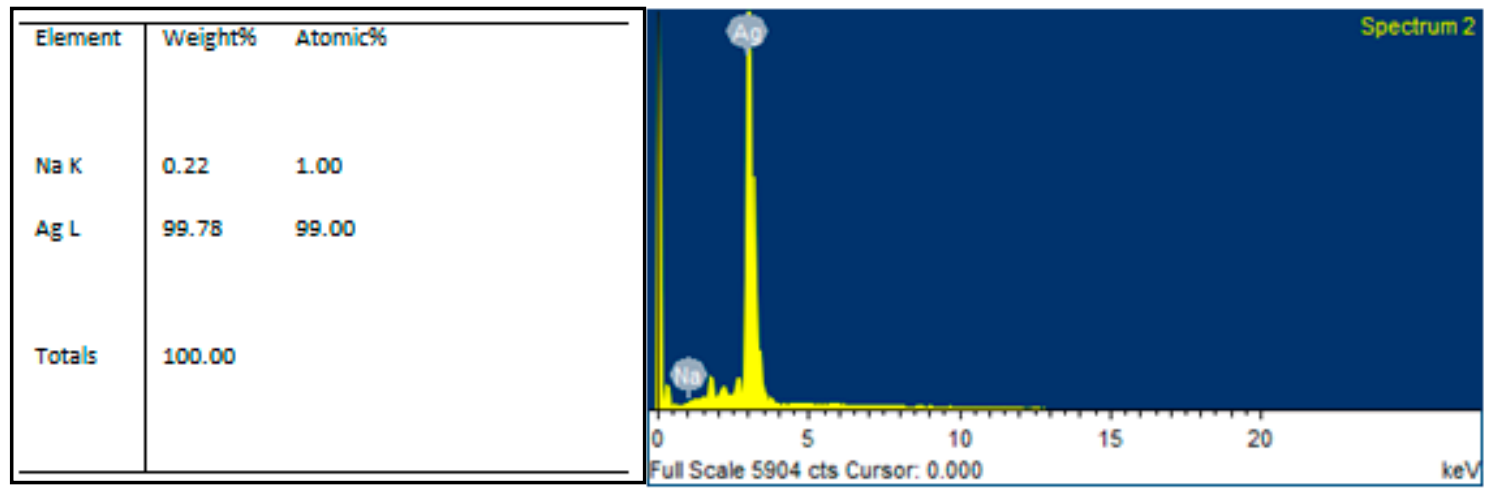

Figure 2. Energy Dispersive Spectroscopy (EDS) analysis of silver nanoparticles.

\subsection{Particle Size Analysis (PSA)}

PSA was done on silver nanoparticles by forming a liquous solution in distilled water. Nanoparticles showed narrow particle size distributions with the majority of particles in the range 50-70 nm (Figure 3).

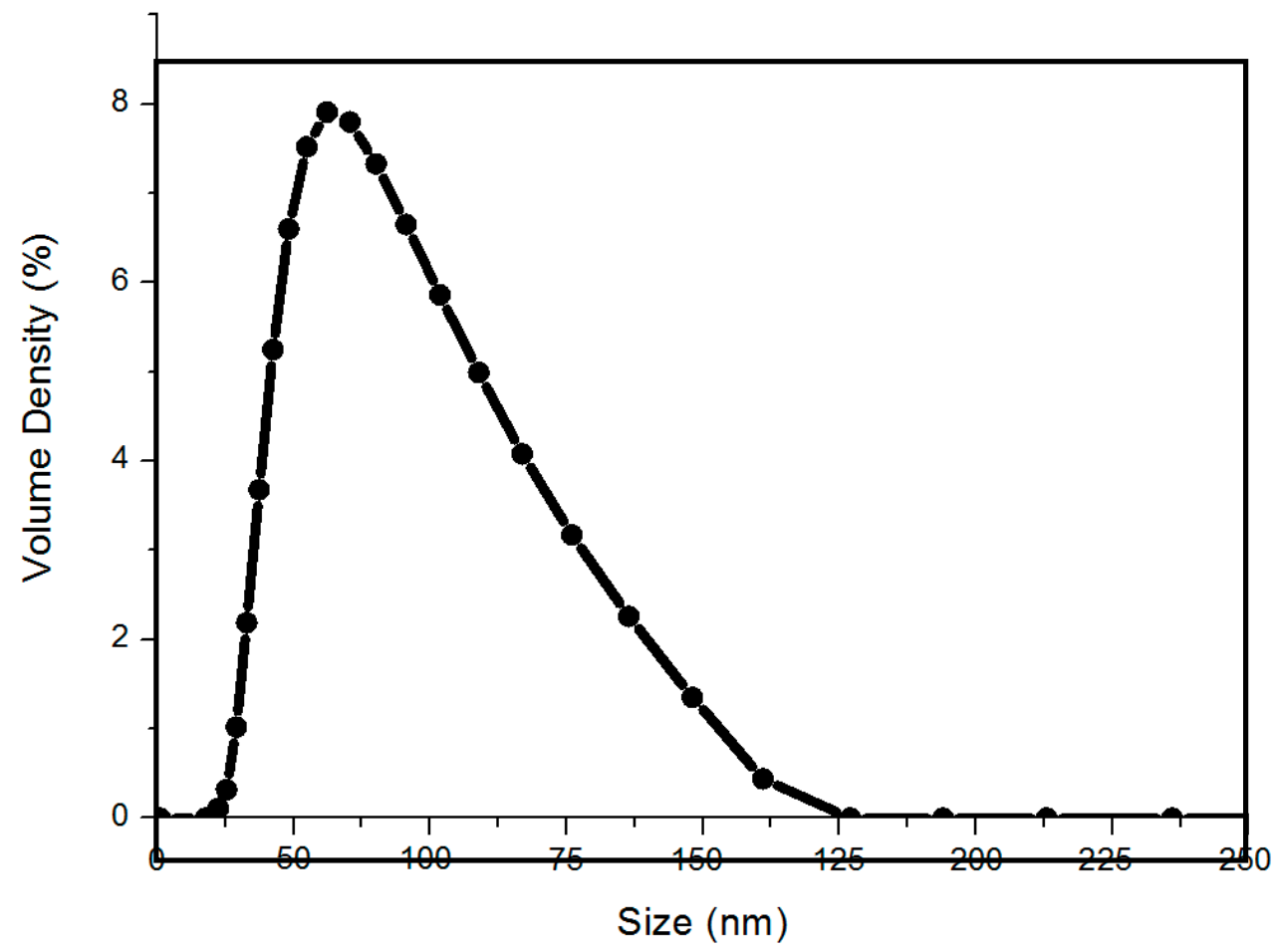

Figure 3. Particle size distribution of silver nanoparticles.

\subsection{Field Emission Scanning Electron Microscopy (FESEM)}

FESEM was performed, during and after the synthesis of silver nanoparticles, to analyze the particle's size and the amount of PEG in the sample as PEG may hinder the electrical properties of the ink. First, the formation of the silver nanoparticles was confirmed. Then, their size and the size distributions were evaluated.

Figure 4 depicts the narrow particle size distribution of as-prepared silver nanoparticles having radii ranging from $54 \pm 2$ to $65 \pm 2 \mathrm{~nm}$. After adding the viscosifying solution and after deposition over the glass substrate by heating at $150^{\circ} \mathrm{C}$, a film was observed under FESEM. The morphology of the conductive film confirmed the linkage between the silver nanoparticles, as shown in Figure 5a,b. 


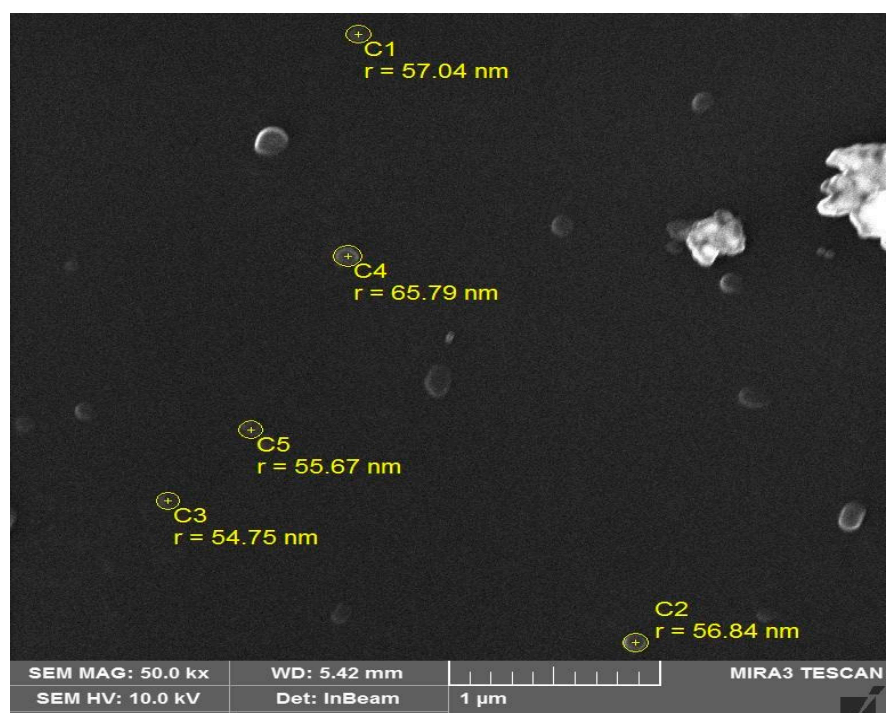

Figure 4. FESEM image of as-synthesized Ag nanoparticles.

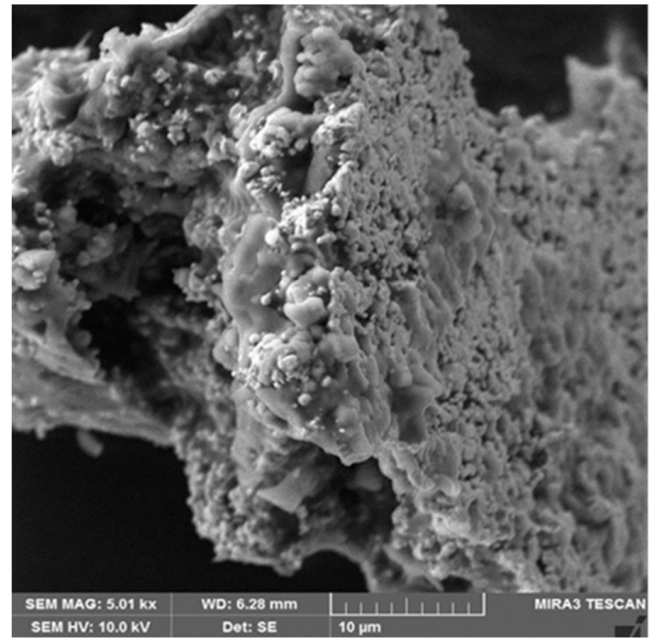

(a)

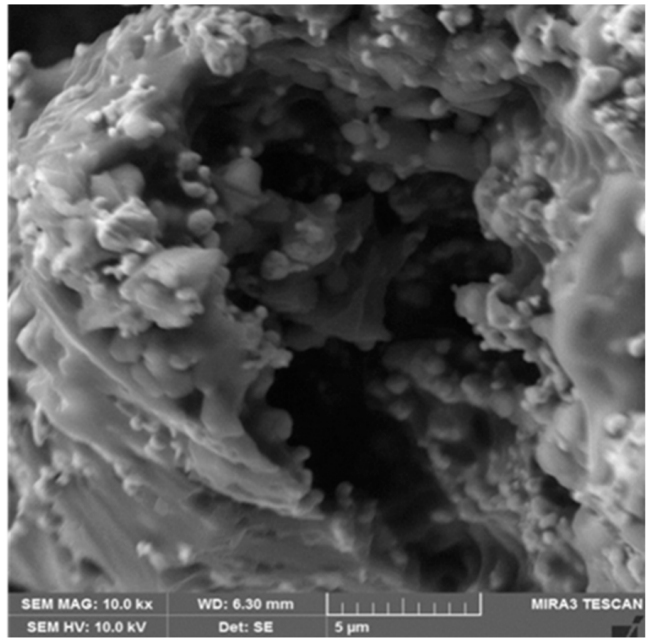

(b)

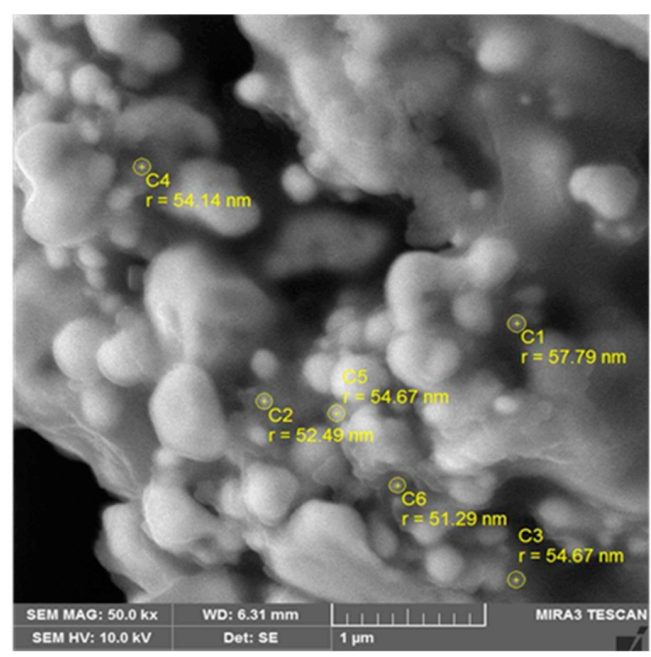

(c)

Figure 5. Microstructure of Ag nanoparticles after adding viscosifying solution: (a) $5.01 \mathrm{kx}$, (b) $10.0 \mathrm{kx}$, and (c) $50.0 \mathrm{kx}$. 
At a higher resolution we found a clearer image of the deposited coating and the structure was easier to interpret. Figure 5b shows the FESEM image of the conductive film at $10 \mathrm{kx}$. In this case, silver nanoparticles of a circular shape were clearly visible and the connection between them was easy to visualize.

In order to find the exact size, the resolution was further enhanced and the film was observed at $1 \mu \mathrm{m}$ (Figure 5c). The particles linked with one another but the viscosifying solution was also seen. The particles' radii were found to range between $51 \pm 2$ and $57 \pm 2 \mathrm{~nm}$ and some particles lost their circular identity after agglomeration with other nanoparticles. Thus, irregular structures were formed.

The samples were permitted to air dry for $20 \mathrm{~min}$. Next, to enhance the linkage between the silver nanoparticles, the sample was heated at 250,400 and $500{ }^{\circ} \mathrm{C}$ for $20 \mathrm{~min}$. As a result of this step the color changed from black to cream (Figure 6) and the conductivity enhanced with the increase in temperature.

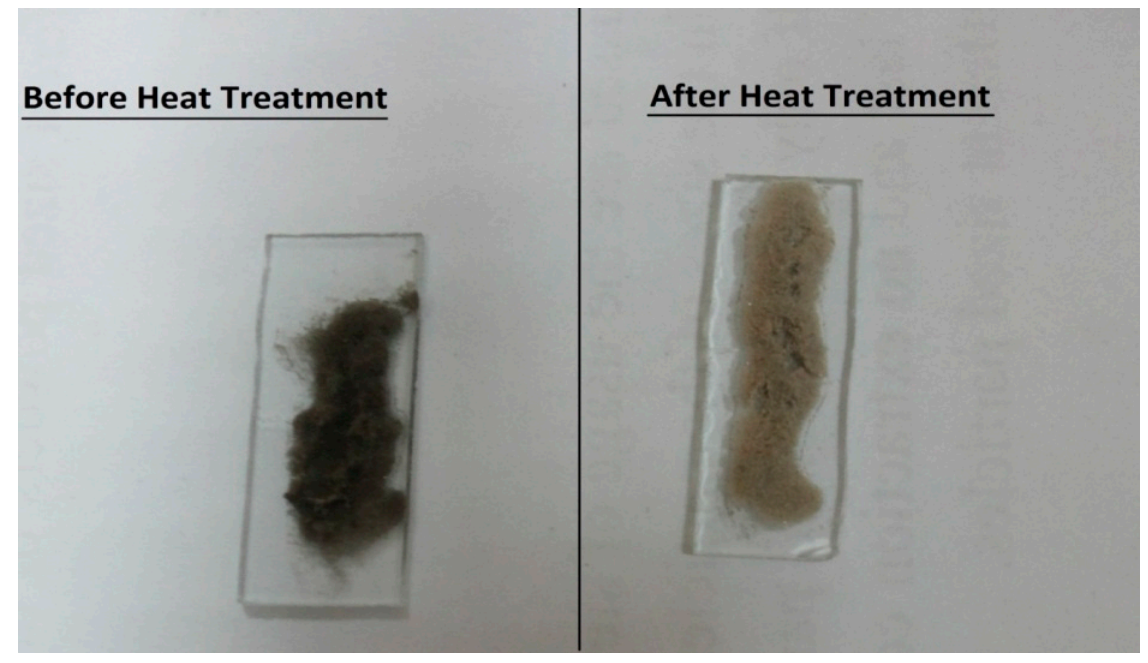

Figure 6. Film before and after heat treatment.

After heating the sample at $500{ }^{\circ} \mathrm{C}$, the interconnectivity between the particles became stronger and the linkages were enhanced along with the conductivity. This structure, shown in Figure 7a, depicts a greater number of particles per unit area compared to Figure 5a. A lesser amount of viscosifying solution was left in this film.

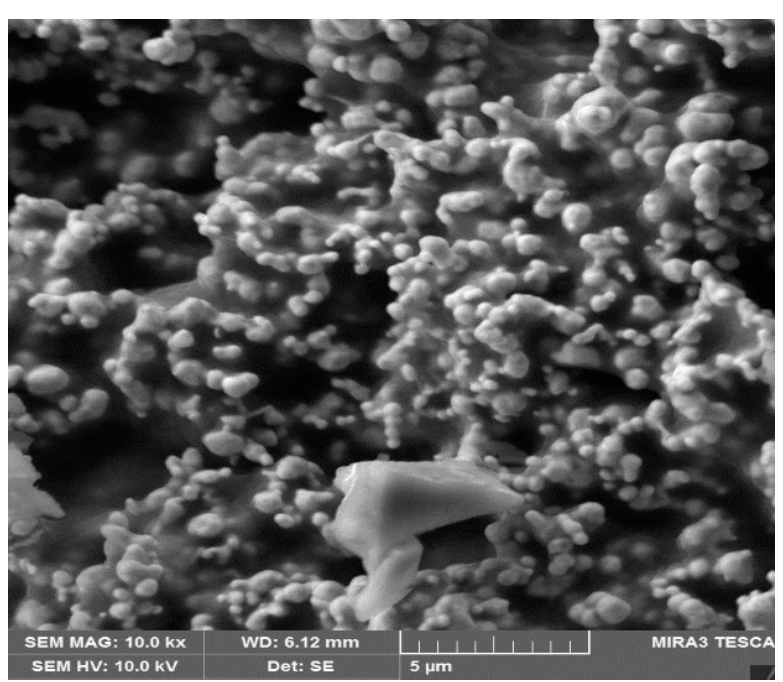

(a)

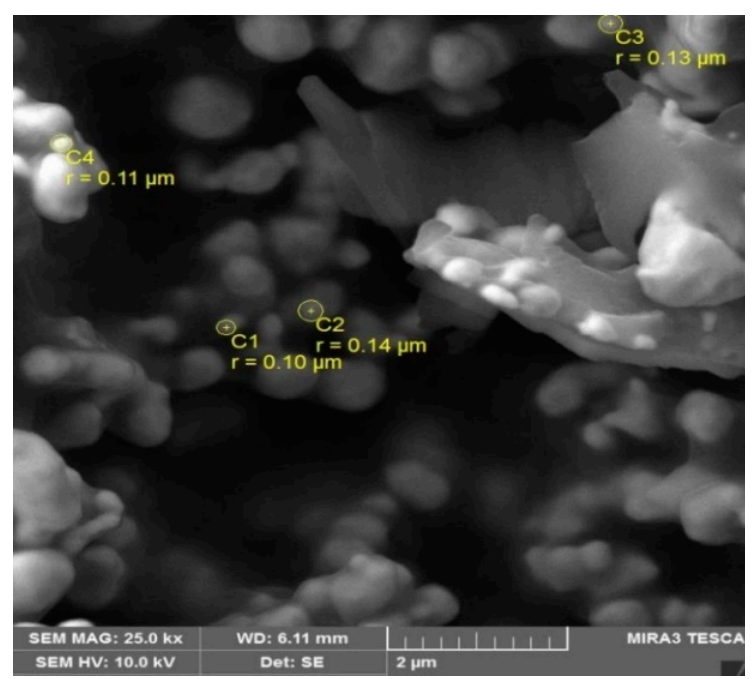

(b)

Figure 7. Cont. 


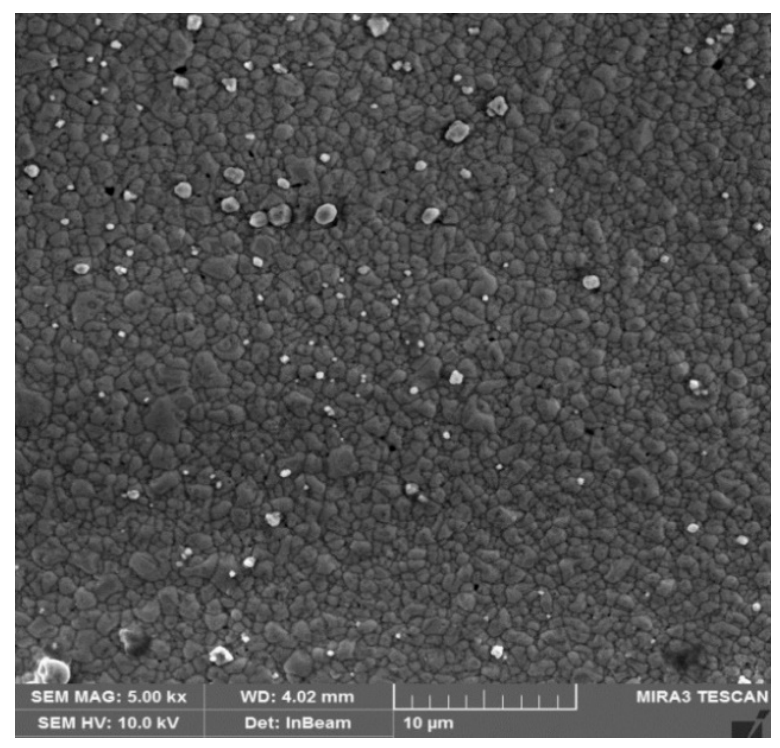

(c)

Figure 7. Microstructure of the conductive film at: (a) $10.0 \mathrm{kx}$, (b) $25.0 \mathrm{kxc}$, and (c) $5.00 \mathrm{kx}$.

The resolution was enhanced to find finer details of the film. Figure $7 \mathrm{~b}$ shows the image at $25.0 \mathrm{kx}$ in which the particles are seen clearly. In this image the radius of the particles ranged from 0.10 to $0.14 \mu \mathrm{m}$. This radius was larger than the radius of the silver nanoparticles, which were heated at $150{ }^{\circ} \mathrm{C}$, and this size incrementation was attributed to nanoparticle agglomeration from heat treatment.

Figure $7 \mathrm{c}$ displays the image of the silver conductive film at $5.00 \mathrm{kx}$. A very clear surface with smooth interconnections was present and the charge carriers flowed properly. This smooth structure played its role in the enhancement of conductivity and reduced the resistivity to a greater extent.

Subsequent to sintering in ambient conditions, the conductive features were composed of a percolated network of silver particles. Upon annealing at modest temperatures, sintering led to both enhanced particle network densification and enhanced electrical performance [16].

\subsection{Fourier Transformed Infrared Spectrometry (FTIR)}

In Figure 8, three major bands could be seen at the different wavenumbers. First, a broad band ranging from 3000 to $3400 \mathrm{~cm}^{-1}$ validated the presence of EG O-H stretching, while the band at $1639.18 \mathrm{~cm}^{-1}$ was attributed to the carbonyl groups emerging from the oxidation of PEG molecules [27]. The $1350.6 \mathrm{~cm}^{-1}$ band corresponded to the carboxylate groups for the formation of $\mathrm{Ag}$ nanoparticles by a reaction with PEG in an aqueous solution. It was also confirmed that the formation of $\mathrm{Ag}$ nanoparticles was achieved via the air oxidation of the oxyethylene groups of PEG, like that observed in the aqueous solution [28]. The Ag-Ag bond stretching appeared below $400 \mathrm{~cm}^{-1}$ but these $\mathrm{Ag}-\mathrm{Ag}$ bonds could not be seen on the FTIR spectrum, which is limited to the mid-infrared region (4000-400 $\mathrm{cm}^{-1}$ ) [29]. However, a comparison of the peak positions, with that of the reducing agent, confirmed a slight shift in the wavenumbers, indicative of the weak bonds that could easily be removed during washing. Overall, these findings were consistent with the previous vibrational molecular spectroscopic investigations [27-29].

\subsection{XRD Analysis}

The XRD technique was used to analyze the crystallographic structure of the conductive film. The carbon X-ray source was used, having a 1.5-1.6 ̊ wavelength. Silver has a face-centered cubic (FFC) crystal structure. Figure 9 shows the XRD graph displaying the three characteristics peaks at $2 \theta$ $=38.121^{\circ}, 44.308^{\circ}$ and $64.458^{\circ}$. These peaks can be indexed as (111), (200) and (220), respectively [17]. 
The calculated crystallite size of silver was $739 \AA$ at constant full width half maximum (FWHM) and the average lattice constant was $4.0855 \AA$.

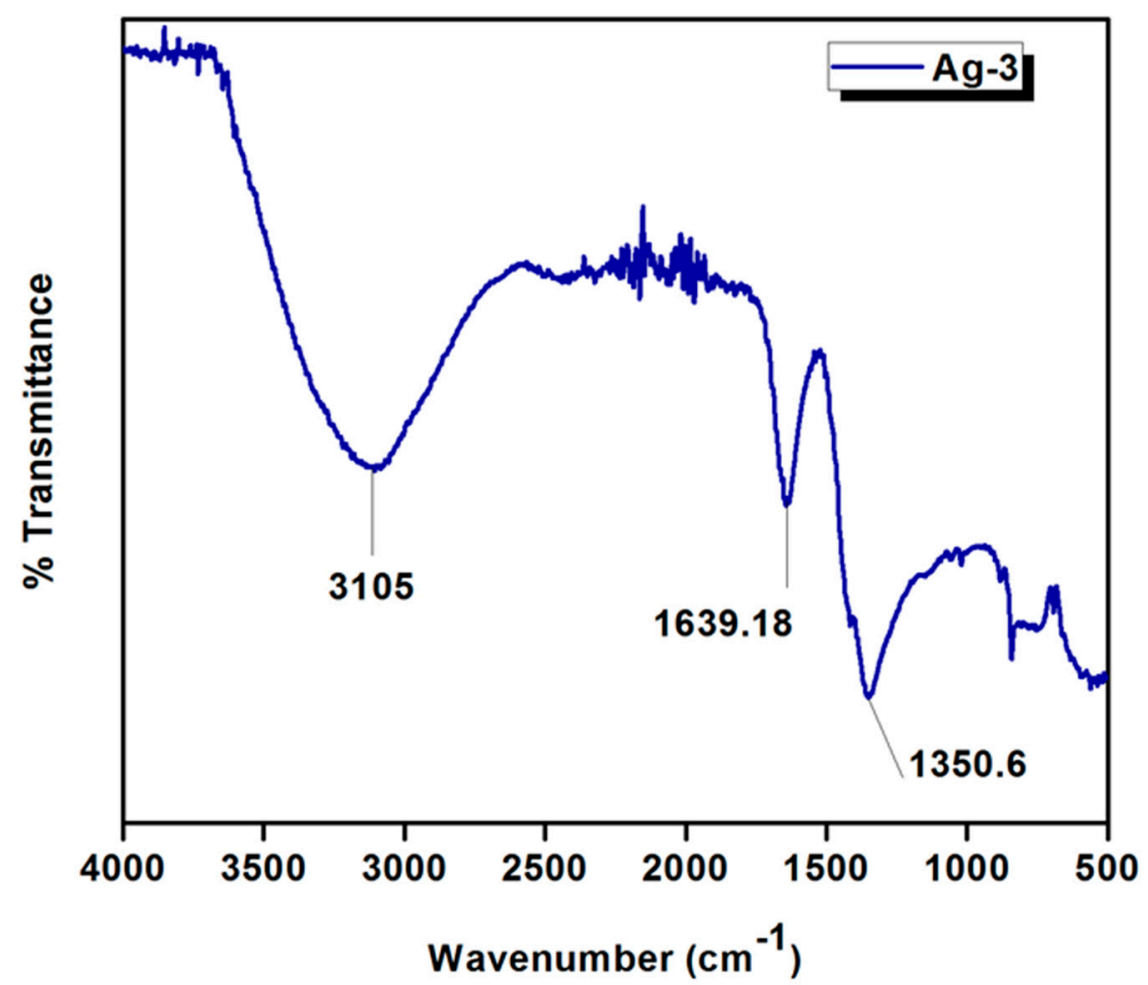

Figure 8. Fourier Transformed Infrared Spectrometry (FTIR) spectrum of conductive film.

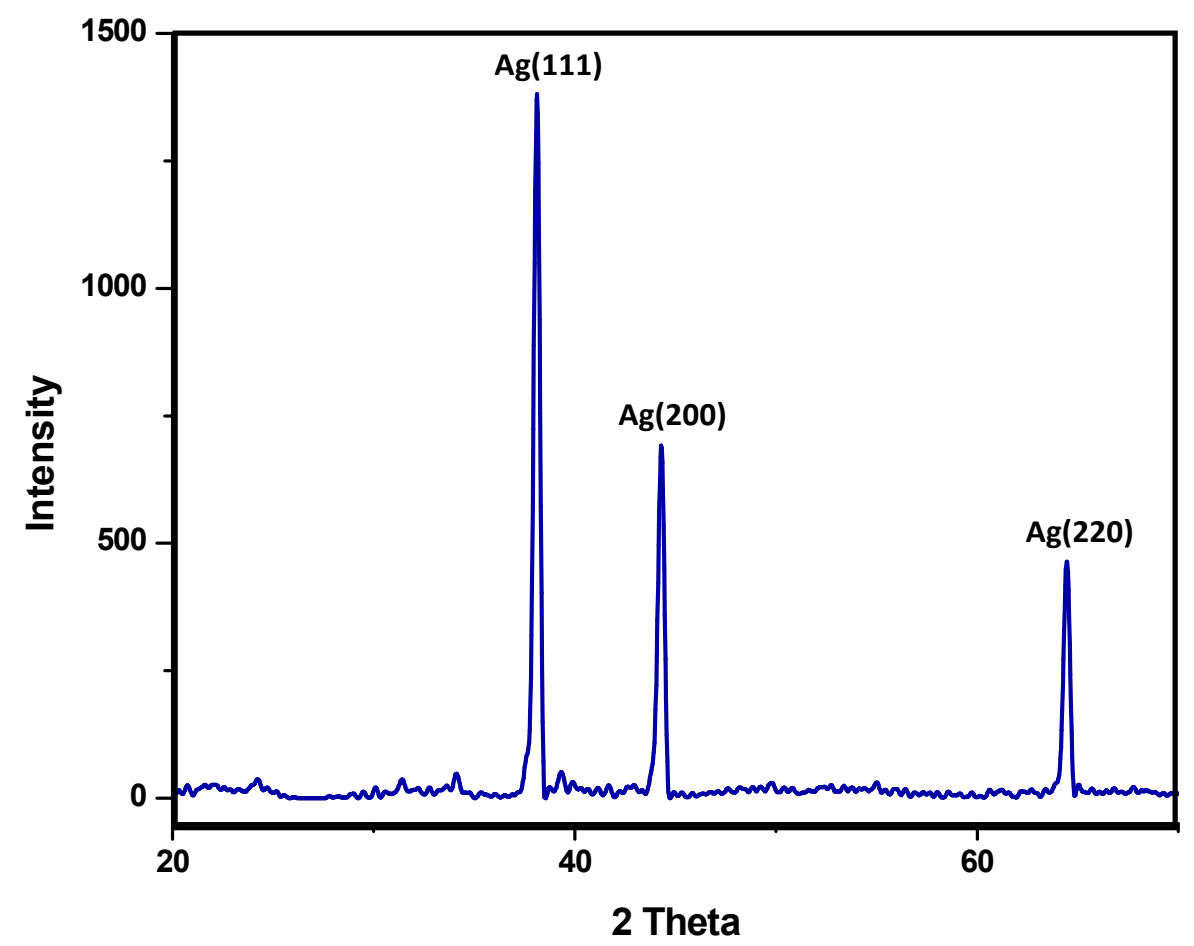

Figure 9. X-ray diffraction analysis of silver conductive film. 
This analysis proved the face centered cubic (FCC) structure of the silver and also indicated the absence of silver oxide, further confirming that there was no oxidation of silver nanoparticles from the capping efficiency of PEG.

\subsection{Physical Properties of Silver Precursor Ink}

The conductive films obtained were stable, viscous enough to give the appropriate fluid flow and surface tension while filming. The major factor in the application of these silver precursor inks is the stability of the conductive film, in terms of conductivity and physical properties. These considerations were dealt with using a viscosifying solution, which also avoided precipitation of the silver.

\subsection{Sintering of Silver Films}

The conductive films were deposited using silver precursor ink. Figure 6 shows the optical images of sintered conductive films at 50 and $500{ }^{\circ} \mathrm{C}$, respectively. The film changed color, from dark greyish to cream, after heat treatment. The silver film clung to the substrate without breaks in the wake of sintering and the initially nonconductive film became conductive $[18,19]$.

\subsection{Temperature Resistivity Correlation of Silver Films}

Resistivity is an intrinsic property and to find the resistivity we first evaluated the resistance using a digital multimeter (DMM) (EDM-4760). After finding the resistance $(\Omega)$ we calculated resistivity using the following Equation (1):

$$
R=\frac{\rho L}{A},
$$

where $\rho$ is resistivity, $\mathrm{R}$ is the resistance, $\mathrm{L}$ is the length of material (distance between two probes of DMM) and A is cross-sectional area of film.

Next, the length and area of the cross-section needed to be calculated. The length was kept at $1 \mathrm{~cm}$ and FESEM was employed to find the area of the cross-section. To estimate the cross-section, the width and thickness of the conductive film were observed by FESEM at 86 times magnification. Three values of the width from different positions were taken and their mean value was calculated. The mean value of the width of the conductive film was $1453.89 \times 10^{-6} \mathrm{~m}$.

To obtain the thickness value we analyzed the cross-section of the film using FESEM. Figure 10 shows the cross-section of the conductive film at $1 \mathrm{kx}$. For a precise value the mean thickness of four measurements was taken, rendering a value of $20.59 \times 10^{-6} \mathrm{~m}$. After obtaining these values we calculated the area by the following equation:

$$
\text { Area of cross-section }(\mathrm{A})=\text { Width } \times \text { Thickness. } \mathrm{A}=2.99 \times 10^{-12} \mathrm{~m}^{2}
$$

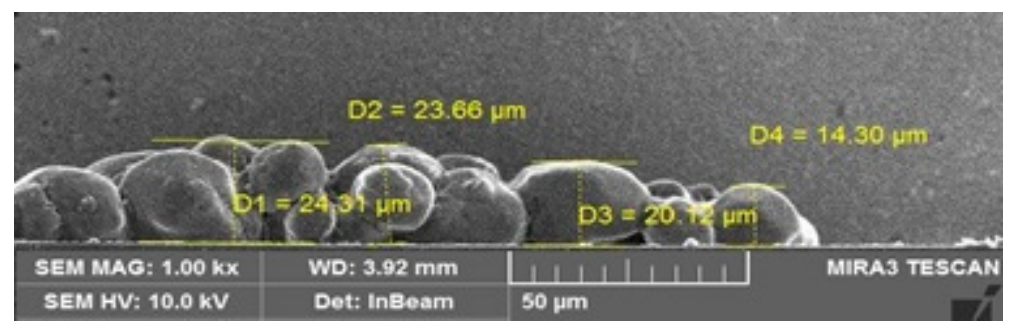

Figure 10. Cross-sectional image of conductive film for thickness measurement.

There was an inverse relationship between resistivity and temperature, demonstrating that by increasing the temperature there was a drop in the resistivity value. Figure 11 shows the same behavior as the value of resistivity diminished and the temperature rose. The minimum resistivity obtained was $0.04485 \times 10^{-6} \Omega \mathrm{m}\left(4.485 \times 10^{-6} \Omega \mathrm{cm}\right)$. Finally, the resistivity value obtained in this research 
was found to be comparable with the previous reports of silver nanofilms approaching bulk silver (about $1.6 \times 10^{-6} \mathrm{ohm} \cdot \mathrm{cm}$ ) $[10,22]$. This behavior can be ascribed to an enhanced agglomeration at elevated temperatures that led to the interconnections required for the charge carriers to flow causing the resistivity value to drop.

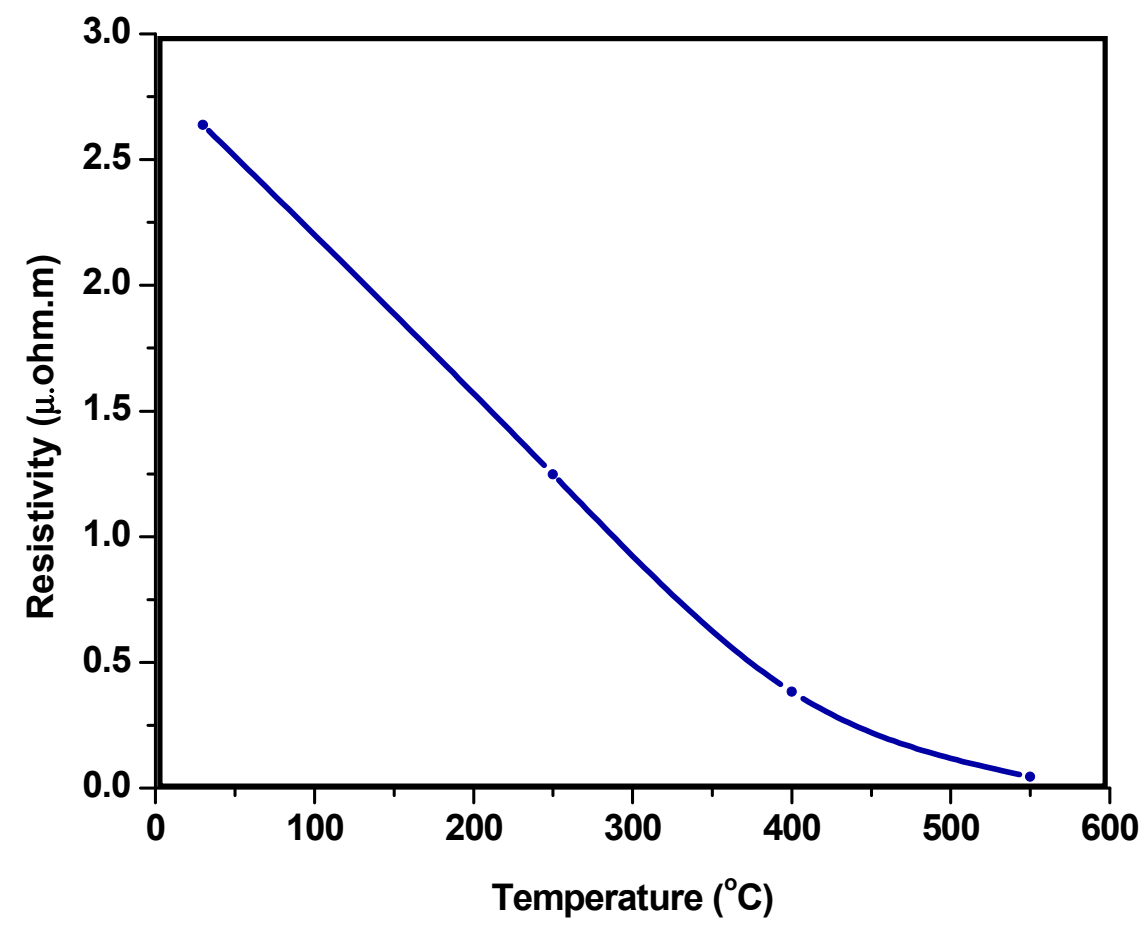

Figure 11. Behavior of resistivity with rise in temperature.

\section{Conclusions}

The conductive silver nanoparticle solution synthesized in our work can be used to produce flexible electrical circuits via simple pattering techniques. Highly dispersed silver nanoparticles were synthesized for printed electronics. A facile approach using a PEG of different molecular weights as surfactant was designed to make a conductive silver solution. The process was rendered cost effective using a simplified synthesis technique and low-cost reducing and capping agents. This is the first report of the synthesis of silver nanoparticles using a combination of silver nitrate and a mixture of three molecular weights of PEG and DEA, which rendered a stable morphology of nanoparticles and enhanced the conductivity as a function of temperature. However, the resistivity was not affected by this synthesis combination. Furthermore, conductive films were deposited on the glass substrate using the viscosifying solution. The conductivity was enhanced with the rise in temperature and was found to be effective in ambient temperature conditions. Future investigations of the optimization of films can improve the conductivity, adhesive properties, viscosity and flexibility to a level applicable to ink jet printers.

Author Contributions: Conceptualization, F.M, M.R, and S.S.; methodology, F.M, M.R, and S.S.; software, F.M.; validation, F.M, M.R, and S.S.; formal analysis, F.M and S.S.; investigation, F.M, and M.R.; resources, S.S.; writing —original draft preparation, F.M.; writing—review and editing, F.M, and S.S.; supervision, S.S.; project administration, S.S.; funding acquisition, S.S.

Funding: This research was funded by the Higher Education Commission (HEC), NRPU project No. 5851/Federal/NRPU/R\&D/HEC/2016 and Institute of Space Technology.

Acknowledgments: S. Shabbir is grateful to the Higher Education Commission (HEC) of Pakistan for providing funds for this work under the NRPU project No. 5851/Federal/NRPU/R\&D/HEC/2016.

Conflicts of Interest: The authors declare no conflicts of interest. 


\section{References}

1. Chen, Y.; Au, J.; Kazlas, P.; Ritenour, A.; Gates, H.; McCreary, M. Flexible active matrix electronic ink display. Nature 2003, 423, 136. [CrossRef]

2. Bairavasubramanian, R.; Thompson, D.; Ponchak, G.E.; Tentzeris, M.M.; Papapolymero-u, J. Liquid crystal polymer (LCP): A new organic material for the development of multilayer dual-frequency/dual-polarization flexible antenna arrays. IEEE Antenn Wirel Pr. 2005, 4, 22-26.

3. Otte, K.; Makhova, L.; Braun, A.; Konovalov, I. Flexible Cu (In, Ga) $S e_{2}$ thin-film solar cells for space application. Thin Solid Films 2006, 511, 613-622. [CrossRef]

4. Baeg, K.J.; Khim, D.; Kim, J.; Yang, B.D.; Kang, M.; Jung, S.W. High-performance top-gated organic field-effect transistor memory using electrets for monolithic printed flexible NAND flash memory. Adv. Funct. Mater. 2012, 22, 2915-2926. [CrossRef]

5. Wang, J.Z.; Zheng, Z.H.; Li, H.W.; Huck, W.T.S.; Sirringhaus, H. Dewetting of conducting polymer inkjet droplets on filmed surfaces. Nature Mater. 2004, 3, 171-176. [CrossRef]

6. Huang, X.; Qi, X.; Boey, F.; Zhang, H. Graphene-based composites. Chem. Soc. Rev. 2012, 41, 666-686. [CrossRef] [PubMed]

7. Mensing, J.P.; Kerdcharoen, T.; Sriprachuabwong, C.; Wisitsoraat, A.; Phokharatkul, D.; Lomas, T. Facile preparation of graphene-metal phthalocyanine hybrid material by electrolytic exfoliation. J. Mater. Chem. 2012, 22, 17094-17099. [CrossRef]

8. Wu, Y.; Li, Y.; Ong, B.S. Printed silver Ohmic contacts for high-mobility organic thin-film transistors. J. Am. Chem. Soc. 2006, 128, 4202-4203. [CrossRef]

9. Choi, C.S.; Jo, Y.H.; Kim, M.G.; Lee, H.M. Control of chemical kinetics for sub-10 nm Cu nanoparticles to fabricate highly conductive ink below $150{ }^{\circ} \mathrm{C}$. Nanotechnology 2012, 23, 065601-065609. [CrossRef]

10. Russo, A.; Ahn, B.Y.; Adams, J.J.; Duoss, E.B.; Bernhard, J.T.; Lewis, J.A. Pen-on-paper flexible electronics. Adv. Mater. 2011, 23, 3426-3430. [CrossRef] [PubMed]

11. Hösel, M.; Krebs, F.C. Large-scale roll-to-roll photonic sintering of flexo printed silver nanoparticle electrodes. J. Mater. Chem. 2012, 22, 15683-15688. [CrossRef]

12. Kim, J.; Kang, S.W.; Mun, S.H.; Kang, Y.S. Facile synthesis of copper nanoparticles by ionic liquids and its application to facilitated olefin transport membranes. Ind. Eng. Chem. Res. 2009, 48, 7437-7441. [CrossRef]

13. Li, Y.; Wu, Y.; Ong, B.S. Facile synthesis of silver nanoparticles useful for fabrication of high-conductivity elements for printed electronics. J. Am. Chem. Soc. 2005, 127, 3266-3267. [CrossRef]

14. Wu, J.T.; Hsu, S.L.C.; Tsai, M.H.; Hwang, W.S. Direct inkjet printing of silver nitrate/poly (N-vinyl-2-pyrrolidone) inks to fabricate silver conductive lines. J. Phys. Chem. C. 2010, 114, 4659-4662. [CrossRef]

15. Gamerith, S. Direct ink-jet printing of Ag-Cu nanoparticle and Ag precursor based electrodes for OFET applications. Adv. Funct. Mater. 2007, 17, 3111-3118. [CrossRef]

16. Wu, Y.; Li, Y.; Liu, P.; Gardner, S.; Ong, R.S. Studies of gold nanoparticles as precursors to printed conductive features for thin-film transistors. Chem. Mater. 2006, 18, 4627-4632. [CrossRef]

17. Chou, K.S.; Lai, Y.S. Effect of polyvinyl pyrrolidone molecular weights on the formation of nanosized silver colloids. Mater. Chem. Phys. 2004, 83, 82-88. [CrossRef]

18. Walker, S.B.; Lewis, J.A. Reactive silver inks for filming high-conductivity features at mild temperatures. J. Am. Chem. Soc. 2012, 134, 1419-1421. [CrossRef]

19. Rickerby, J.; Simon, A.; Jeynes, C.; Morgan, T.J.; Steinke, J.H.G. 1,1,1,5,5,5-Hexafluoroacetylacetonate copper(I) poly(vinylsiloxane)s as precursors for copper direct-write. Chem. Mater. 2006, 18, 2489-2498. [CrossRef]

20. Chen, S.; Carroll, D.L. Silver nanoplates: size control in two dimensions and formation mechanisms. J. Phys. Chem. B 2004, 108, 5500-5506. [CrossRef]

21. Szczech, J.B.; Megaridis, C.M.; Gamota, D.R.; Zhang, J. Fine-Line Conductor Manufacturing Using Drop-On-Demand PZT Printing Technology. IEEE Trans. Electron. Packag. Manuf. 2002, 25, 26-30. [CrossRef]

22. Adams, J.J.; Duoss, E.B.; Malkowski, T.F.; Motala, M.J.; Ahn, B.Y.; Nuzzo, R.G.; Bernhard, J.T.; Lewis, J.A. Conformal Printing of Electrically Small Antennas on Tree-Dimensional Surfaces. Adv. Mater. 2011, 23, 1335-1340. [CrossRef] 
23. Huang, Q.; Shen, W.; Song, W. Synthesis of colourless silver precursor ink for printing conductive films on silicon nitride substrates. Appl. Surf. Sci. 2012, 258, 7384-7388. [CrossRef]

24. Lopatina, L.I.; Sergeyev, V.G. The effects of the molecular weight and structure of poly(acrylic acid) on the formation of "blue silver". Moscow Univ. Chem. Bull. 2010, 65, 331-334. [CrossRef]

25. Sharmeli, K.; Ahmad, M.B.; Jazayeri, S.D.; Sedaghat, S.; Shabanzadeh, P.; Jahangirian, H.; Mahdavi, M.; Abdollahi, Y. Synthesis and characterization of polyethylene glycol mediated silver nanoparticles by the green method. Int. J. Mol. Sci. 2012, 13, 6639-6650. [CrossRef]

26. Song, Y.J.; Wang, M.; Zhang, X.-Y.; Wu, J.-Y.; Zhang, T. Investigation on the role of the molecular weight of polyvinyl pyrrolidone in the shape control of high-yield silver nanospheres and nanowires. Nanoscale Res. Lett. 2014, 9, 17-25. [CrossRef] [PubMed]

27. Dong, H.C.; Yan, W.H. Spontaneous formation of Ag nanoparticles in dimethylacetamide solution of poly(ethylene glycol). J. Colloid Interface Sci. 2002, 255, 299-302.

28. Longenberger, L.; Mills, G. Formation of metal particles in aqueous solutions by reactions of metal complexes with polymers. J. Phys. Chem. 1995, 99, 475-478. [CrossRef]

29. Gharibshahi, L.; Saion, E.; Gharibshahi, E.; Shaari, A.H.; Matori, K.A. Structural and optical properties of Ag nanoparticles synthesized by thermal treatment method. Materials 2017, 10, 402. [CrossRef] [PubMed]

(C) 2019 by the authors. Licensee MDPI, Basel, Switzerland. This article is an open access article distributed under the terms and conditions of the Creative Commons Attribution (CC BY) license (http://creativecommons.org/licenses/by/4.0/). 